\title{
Labyrinthe
}

$6 \mid 2000$

Numéro 6

\section{Les femmes célèbres sont-elles des grands hommes comme les autres?}

Contribution à l'étude de la statuomanie parisienne

\section{Christel Sniter}

\section{OpenEdition}

\section{Journals}

Édition électronique

URL : http://journals.openedition.org/labyrinthe/456

DOI : $10.4000 /$ labyrinthe.456

ISSN : 1950-6031

\section{Éditeur}

Hermann

\section{Édition imprimée}

Date de publication : 1 juin 2000

Pagination : 51-72

\section{Référence électronique}

Christel Sniter, «Les femmes célèbres sont-elles des grands hommes comme les autres ?»,

Labyrinthe [En ligne], 6 | 2000, mis en ligne le 11 mai 2005, consulté le 01 mai 2019. URL : http:// journals.openedition.org/labyrinthe/456 ; DOI : 10.4000/labyrinthe.456

\section{Ce document a été généré automatiquement le 1 mai 2019.}

Propriété intellectuelle 


\title{
Les femmes célèbres sont-elles des grands hommes comme les autres?
}

\author{
Contribution à l'étude de la statuomanie parisienne
}

\section{Christel Sniter}

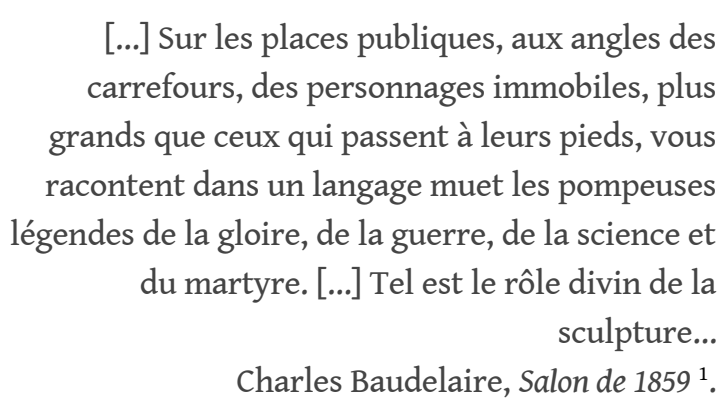

1 Si de nos jours la statuaire publique héritée $d u x^{e}{ }^{e}$ siècle constitue un élément quasi invisible du paysage urbain, elle apparaissait au contraire sous la III République comme un ensemble structurant de l'identité d'une ville. Entre 1870 et 1914, Paris fut témoin de l'apogée de la «statuomanie » : 150 statues $^{2}$ dédiées à des personnages célèbres y sont érigées, contre 26 entre 1815 et 1870 .

2 La floraison de statues de Grands hommes répondait à un triple enjeu, esthétique, politique et social. On voulait orner une ville réorganisée en profondeur par les travaux $\mathrm{du}$ baron Haussmann sous le Second Empire, fortifier le nouveau régime par la glorification des personnages de l'Histoire de France qui incarnaient les valeurs et les combats de la République et refléter un modèle social fondé sur le mérite personnel et non sur la naissance, conformément aux conceptions individualistes de la bourgeoisie, parvenue au pouvoir ${ }^{3}$.

3 Support stable et " économique ${ }^{4}$ », la statue fait de Paris un panthéon à ciel ouvert. Elle apparaît comme un point de jonction matériel entre le passé mythique des grands précurseurs de la Révolution et un futur républicain, qui gardera en mémoire les valeurs qu'incarnent ses héros et que transmettent les statues. Entre filiation historique et construction de la trace, elles s'intègrent au présent comme un puissant moyen symbolique d'instruction civique, d'édification morale et de mobilisation des Français. 
4 La statuaire publique, relativement peu étudiée ${ }^{5}$ jusqu'à maintenant, mériterait d'être constituée en objet de la science politique. De sa conception par les commanditaires à la commémoration qu'elle suscite, la statue, objet intermédiaire entre l'œuvre d'art et le monument public, constitue un point de cristallisation, un repère signifiant où vont éclore certains comportements, s'affirmer certaines positions, qu'elles soient politiques (par exemple les affrontements entre républicains et nationalistes autour des statues de Jeanne d'Arc) ou sociales (les rassemblements féministes devant le monument dédié à Maria Deraismes). Totem ou trophée, la statue dans l'espace public est créatrice de lien social et/ou révélatrice de tensions, dans le cadre de la lutte pour le monopole de la production du sens. «En tant qu'objet symbolique, [elle] condense des projections sur un matériau brut, dès lors investi de sens ${ }^{6} »$.

5 À cette problématique générale de la statue comme pôle de construction identitaire se superposent les questions spécifiques aux statues de femmes célèbres. Parmi les 150 statues érigées à Paris à cette période, onze projets sont dédiés à des femmes : on honore Jeanne d'Arc (à quatre reprises), George Sand, la comtesse de Ségur, Maria Deraismes, Mesdames Hirsch et Boucicaut et l'on tente d'honorer Madame de Staël, Clémence Royer et Louise Michel7 .

6 Pour la première fois dans l'histoire de France, des femmes sont publiquement glorifiées, individuellement, en tant que personnes et officiellement reconnues comme actrices historiques et civiques ${ }^{8}$. Ces mises en scènes inédites de la femme semblent radicalement trancher avec sa représentation traditionnelle dans l'art public et avec sa place dans la société, ses fonctions publiques se cantonnant au philanthropisme.

7 On peut donc légitimement s'interroger sur les causes à l'origine de ces statues qui placeraient de fait les femmes dans la posture masculine de l'acteur national et du Grand homme. Serait-ce un effet accidentel de l'engouement statuomaniaque ou, à l'opposé, la conséquence de l'essor des mouvements féministes qui connaissent à cette époque un véritable âge d'or ? La réponse est sans doute plus complexe, dans la mesure où ce phénomène ne peut être réduit ni à un hasard ni à une action militante. L'analyse des commanditaires, du site, de l'esthétique et de la production du sens (cérémonie d'inauguration et commémorations) de ces statues permet d'éclairer et de nuancer dans le même temps une série de distinctions entre hommes et femmes et entre les femmes elles-mêmes sur le double plan des commanditaires et des statues. S'intéresser aux statues de femmes par rapport aux statues d'hommes permet de mettre en valeur une expérience de la mixité entre hommes et femmes à la fois comme objets statufiés et comme acteurs au sein des comités privés qui, bien souvent, ont pris l'initiative du projet monumental ${ }^{9}$.

8 Face à cette incongruité parmi le peuple des statues de pierre et de bronze de la capitale, plusieurs questions émergent. Ces femmes célèbres sont-elles traitées sur le même mode que les hommes ? En quoi leur représentation diffère-t-elle de la représentation traditionnelle de la femme dans l'espace public et dans la société ? Peut-on même établir une différence de traitement parmi les statues de femmes célèbres entre une Jeanne d'Arc, personnage à haute teneur symbolique et d'autres femmes moins héroïsées ?

9 Si l'on tente de saisir la spécificité des statues de femmes à travers le prisme de la " différence ", plusieurs angles d'analyses peuvent être distingués. Les statues de femmes tranchent avec la représentation traditionnelle de la femme dans l'art statuaire et avec la conception de la femme dans la société de l'époque. Des différences existent entre les 
statues de femmes célèbres et sont encore plus importantes si on les compare aux statues d'hommes célèbres.

L'AVÈNEMENT DES « GRANDES CONTEMPORAINES » Une représentation inédite de la femme

Force est de constater que la présence de la femme dans le peuple des statues parisiennes se manifeste dans une énorme majorité des cas ${ }^{10}$ par l'utilisation de son corps pour évoquer des notions abstraites ou comme support décoratif.

11 L'usage de l'allégorie, forme d'utilisation du corps féminin inspirée de l'Antiquité, se développe à la fin du $\mathrm{xIX}^{\mathrm{e}}$ siècle. Dans l'ornementation intérieure et extérieure des édifices publics, accolées à un monument, les grandes vertus - la Justice, la Liberté, la Renommée, mais aussi la Nation, l'Industrie, le Commerce, l'Agriculture - sont personnifiées par des femmes. Marianne constitue un cas particulier. Symbole de la République, il s'agit d'une allégorie et non d'un individu, elle incarne la continuité d'un régime politique dont les personnalités au pouvoir étaient par trop changeantes ${ }^{11}$. Mais cette représentation féminine n'a été sans doute possible que parce que les femmes étaient exclues $d u$ vote $^{12}$ et renforçait même cette exclusion, imposant une image abstraite de la femme et rendant ainsi plus difficile la conception d'une femme ordinaire citoyenne.

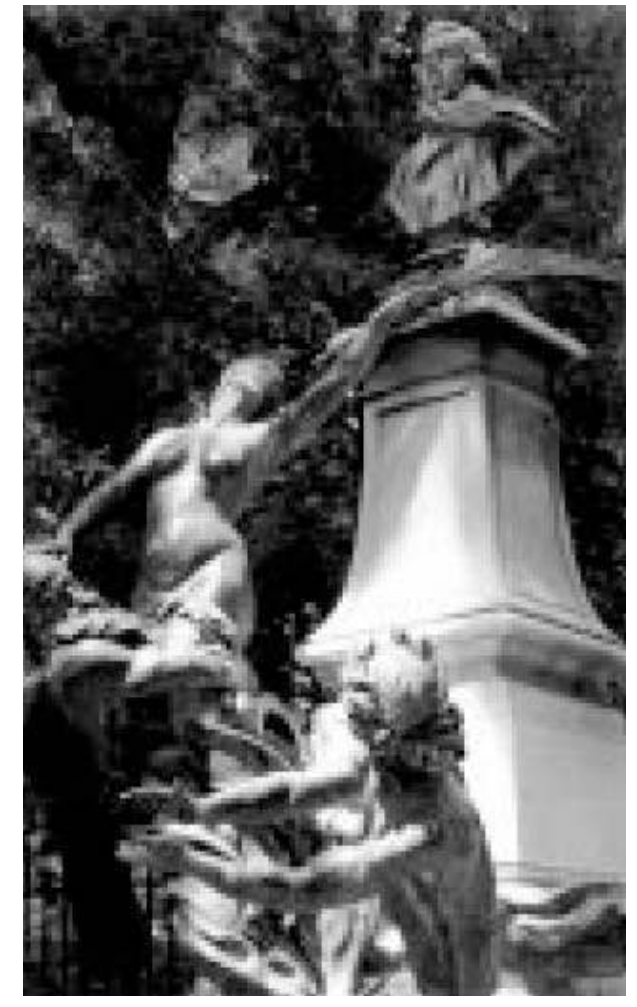

III. 1 - Monument d'Eugène Delacroix, Dalou, 1890, Jardin du Luxemboug (Tous droits réservés)

Une autre illustration de l'utilisation du corps féminin, non plus comme abstraction mais comme objet : le nu 1900 est le reflet d'une société bourgeoise dominée par les hommes. Les cariatides, les femmes-lampadaires ${ }^{13}$, les femmes-fontaines sont autant d'objectivations du corps de la femme. Ce phénomène est facilité par l'art industriel et les progrès de reproduction des œuvres grâce aux techniques de la « mise au point ${ }^{14}$ » et de la fonte « à la cire perdue $\aleph^{15}$. Les monuments dédiés aux grands hommes sont souvent accompagnés 
d'une allégorie, muse inspiratrice, comme les monuments dédiés à Delacroix (ill. 1) ou à Fauré, ou encore d'un personnage féminin fictif, comme le monument à Maupassant ${ }^{16}$. Maurice Agulhon explique cette instrumentalisation multiple de l'image de la femme :

Le $\mathrm{XIX}^{\mathrm{e}}$ siècle bourgeois répand et banalise une culture d'humanités classiques, où tout bachelier, pétri de latin et de mythologie, connaît les Déesses et les Vertus, leurs attributs et leurs costumes; et ce même XIX siècle bourgeois se donne par ailleurs des mœurs et une mentalité masculines, voire (si l'on peut dire) masculinistes, qui se complaisent dans l'exhibition et la contemplation voluptueuse de l'image de la femme-objet. [...] Les rues s'emplissent de belles femmes, sculpturales - c'est le cas de le dire - et inexpressives, drapées à l'antique, ou dénudées, ou voilées à demi, et dont l'allure [...] fait de toute façon contraste avec le réalisme moderne et expressif des grands hommes, qui sont presque toujours des hommes ${ }^{17}$.

C'est précisément ce " presque » qui nous intéresse en ce qu'il tranche nettement avec ces images réificatrices et réductrices de la femme. Les femmes célèbres sont représentées avec réalisme, en habit d'époque (ill. 2), les attributs féminins n'étant pas autant mis en relief. Les statues de femmes célèbres, pour la première fois dans l'histoire, ne représentent pas ces femmes pour leur corps mais bien pour leur personne.

Les statues de femmes célèbres, érigées en modèle, semblent en opposition avec la conception de la femme à l'époque. Quelle image les hommes de la III ${ }^{\mathrm{e}}$ République en ontils ? L'article « femme » du Grand dictionnaire universel du XIX ${ }^{e}$ siècle de Pierre Larousse permet d'en dessiner les contours. Celle-ci est d'abord définie par son appartenance au règne animal et sa fonction reproductrice : « Femelle de l'homme, être humain organisé pour concevoir et mettre au monde des enfants ${ }^{18}$ ». La femme serait restée un enfant, dépendante de ses passions et peu douée de raison ${ }^{19}$. L'obstacle majeur à l'entrée de la femme en République, outre sa dépendance naturelle, est son manque d'éducation.

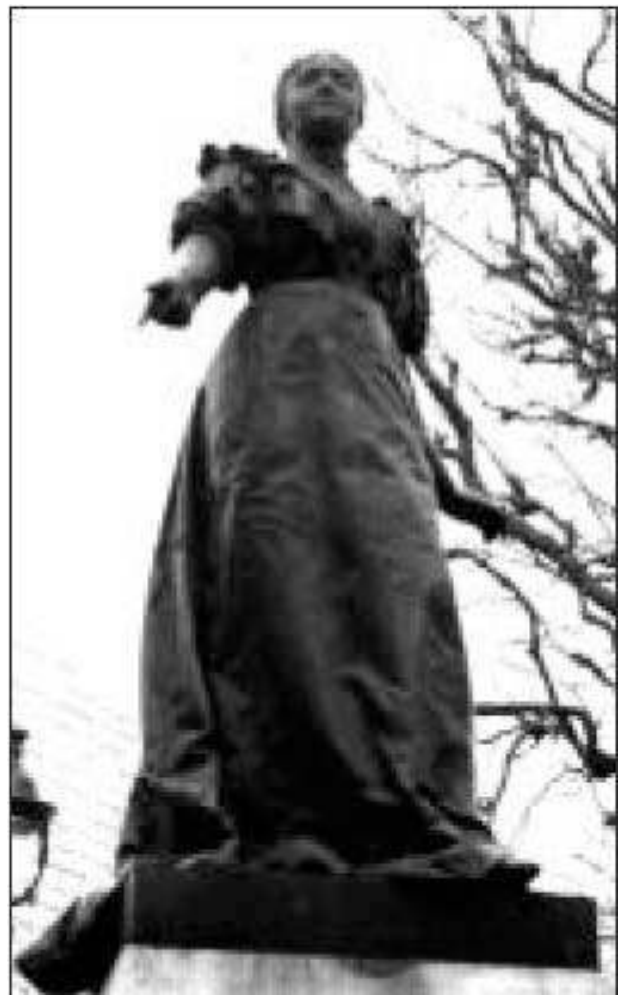


III. 2 - Statue de Maria Deraismes, Barrias, 1898, square des épinettes (Source privée) des meilleurs citoyens de la République alors que les femmes sont si peu considérées dans la société. Comment les producteurs de sens de l'époque ont-ils géré ce paradoxe ? Voyons plus précisément ces représentations inédites et tentons de déceler les différences de traitement qui peuvent exister entre elles selon leur fonction et selon ce qu'elles incarnent.

Un traitement différencié des femmes célèbres places ? L'étude de la symbolique urbaine permet de mesurer l'importance accordée à telle ou telle effigie féminine. L'analyse esthétique dévoile les qualités sélectionnées parmi les multiples facettes d'un personnage et l'image de la femme célèbre ainsi produite.

18 Les statues de femmes de lettres ou de féministes sont érigées à l'initiative de comités privés alors que celles de Jeanne d'Arc sont érigées alternativement par l'État et la Ville de Paris. Le cas de Jeanne d'Arc est unique et semble faire l'unanimité : quatre statues lui sont érigées en un quart de siècle : en 1874, l'État commande la statue de la place des Pyramides au sculpteur Frémiet ; en 1891, la Ville de Paris érige la sienne, à la demande des habitants du quartier, boulevard Saint-Marcel ; vers 1894 est érigée celle de la rue de la Chapelle; en 1900, l'État en érige une seconde place Saint-Augustin.

19 Les autres statues sont érigées après 1900, sauf pour Maria Deraismes dont le monument est érigé en 1898. Toutes résultent d'une initiative privée. Résultat du narcissisme d'une classe bourgeoise dominante, à peine une personne célèbre est-elle morte qu'on lui érige une statue. Dans quatre cas, la mort récente du modèle sert de catalyseur au projet ${ }^{22}$. Si la durée de la procédure est rapide pour Maria Deraismes (quatre années), quatorze ans d'efforts pour Clémence Royer n'aboutissent finalement à rien. L'argument statuomaniaque est souvent mobilisé pour motiver un refus, mais peut cacher des oppositions d'ordre politique, comme dans le cas de Louise Michel, personnage trop à gauche pour un conseil municipal passé à droite.

20 L'analyse des commanditaires de la statuaire publique féminine fait ressortir l'existence d'une collaboration étroite entre hommes et femmes autour d'un projet commun. L'érection d'une statue est un des rares moments dans la vie publique où hommes et femmes font l'expérience de la mixité. Ils mènent à bien une entreprise sociale, de sa conception à sa réalisation. Des intellectuels, hommes politiques, féministes et femmes des salons créent un comité, ouvrent une souscription, rassemblent des fonds et démarchent auprès du conseil municipal afin d'obtenir un site. Par exemple dans le comité de Clémence Royer, sur les 103 membres du comité originel ${ }^{23}$, $36 \%$ sont des femmes, ce qui est la proportion la plus importante de tous les comités connus. Quatre- 
vingt-dix-sept membres sur 103 ont pu être identifiés : 24 députés de la Seine, conseillers municipaux, sénateurs ou maires ; 5 membres éminents du ministère de l'Instruction Publique et des Beaux-Arts ; 13 hommes ou femmes de science ; 23 hommes de lettres et artistes ; 10 femmes de lettres ; 22 membres de sociétés et journaux féministes. La mixité s'affiche d'autant plus lors de la cérémonie d'inauguration où hommes et femmes prononcent des discours, se tiennent côte à côte dans l'assistance, bref agissent ensemble publiquement.

Le choix du site revient au conseil municipal. En quoi peut-il constituer un enjeu et quelle est la fonction urbaine de la statue ? La statue la plus centrale et la plus visible aura le plus d'impact. Celle-ci structure l'espace et lui donne un sens : un centre et une périphérie ; elle est un repère, un point d'aboutissement visuel. La délimitation du site par le socle et souvent une grille basse crée un espace sacré, séparé. Souvent proche d'un lieu où la personne statufiée a vécu, le site devient un lieu de dévotion et de pèlerinage, propice au rassemblement. Les statues érigées dans les jardins ont une fonction moins solennelle. Cependant, une statue comme celle de George Sand au Luxembourg, constitue un repère pour le promeneur qui reconnaît l'espace naturel autour d'elle et les allées anonymes qui y mènent. Celui-ci y associe le nom de George Sand. En ce sens, la statue est bien un repère urbain. Elle permet de donner à l'espace un visage, un nom. Les sites des statues de femmes dévoilent une dichotomie nette : les statues de Jeanne d'Arc, marques des pouvoirs publics, sont toutes érigées sur des places publiques ou dans la rue - elles ont une fonction de repère national ou local ${ }^{24}$ - alors que les autres statues de femmes sont placées dans des espaces verts, squares ou jardins ${ }^{25}$. Les statues de Jeanne d'Arc ponctuent l'espace de circulation, créant des perspectives alors que les femmes de lettres ont une fonction plus intimiste, le square étant un lieu de déambulation, de promenade plus propice à la rêverie.

L'analyse de l'esthétique des statues révèle des différences majeures entre les statues de femmes. Trois des statues de Jeanne d'Arc sont en bronze, matériau cher et prestigieux, permettant de sculpter avec finesse et précision, alors que les autres femmes sont en marbre. Seule exception, la statue de Maria Deraismes, qui est elle aussi en bronze ${ }^{26}$. Dans le groupe des statues de Jeanne d'Arc, celle-ci est représentée en armure, un étendard ou une épée à la main, dans deux des cas à cheval (place des Pyramides et place SaintAugustin). À part les cheveux longs de la statue de Frémiet (place des Pyramides), les attributs de la féminité sont relativement absents de ces représentations de la Pucelle. On aurait pu la représenter agenouillée, entendant des voix, en prière ou sur le bûcher, mais on a choisi une attitude moins chrétienne plus imposante et plus. offensive pour incarner des valeurs patriotiques fédératrices dans le cadre du rétablissement national après la défaite de 1870. Elle incarnerait davantage la France et la résistance à l'étranger. 


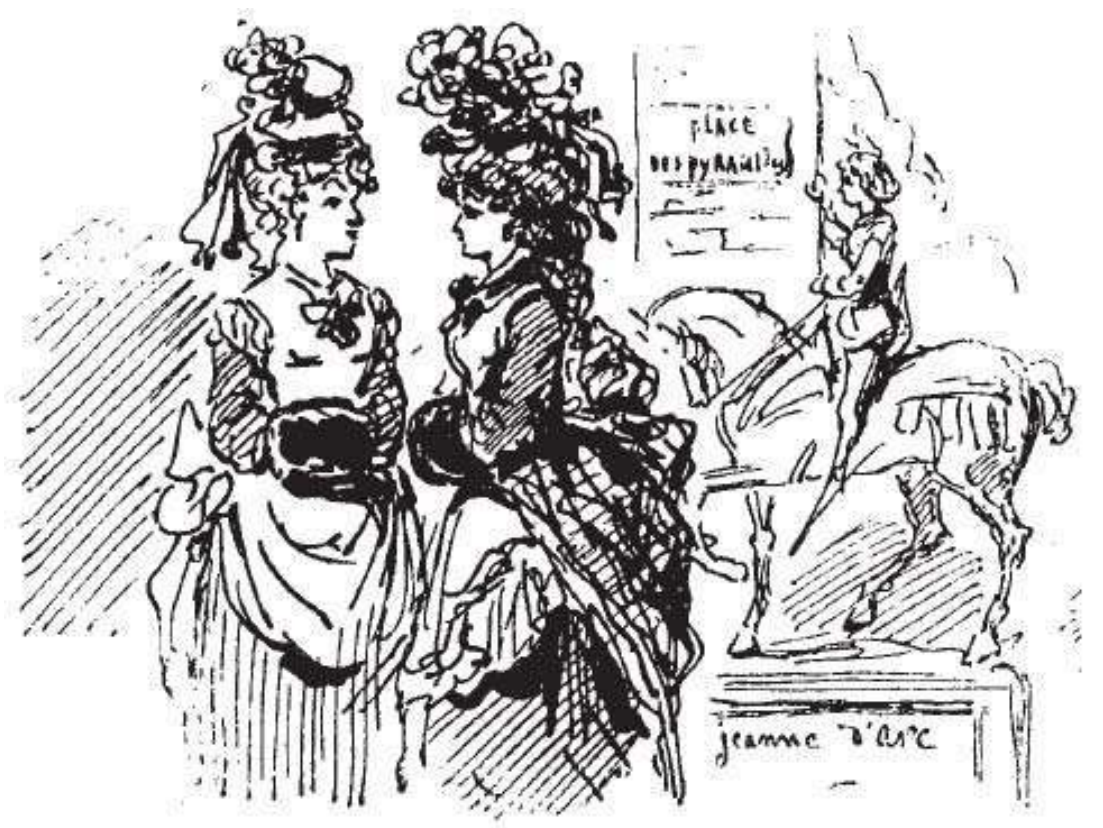

- La vertu, ma clière, vù que ya mene: .

- Place des P'yramides!

III.3 - Dessin humoristique. Source : BHVP, Actualités, Le Monde illustré, 4 avril 1874

Cependant, si on se penche sur les discours et les caricatures de l'époque, elle n'est pas exclusivement vue comme un symbole abstrait mis au service de la République. Elle incarne également une vision de la femme pure et pieuse (ill. 3). On est surpris de voir certaines caricatures de l'époque faire de Jeanne d'Arc non plus un symbole déréalisé mais bien un modèle de féminité auquel les femmes bourgeoises doivent s'identifier. Cette facette féminine de Jeanne d'Arc, évidence oubliée, a été peu mise en valeur.

L'autre groupe est constitué de femmes de lettres (la comtesse de Ségur, George Sand et Madame de Staël), de savantes féministes (Maria Deraismes et Clémence Royer), et de femmes charitables (Mesdames Hirsch et Boucicaut). Si Jeanne d'Arc est représentée davantage comme un homme, les autres femmes apparaissent sous des traits féminins et ce, même quand elles auraient pu être représentées en habits d'homme. Ainsi George Sand n'est pas mise en scène sous les traits du bas-bleu tant décrié par Barbey d'Aurevilly - en habit d'homme et fumant la pipe - mais dans une belle robe, avec une coiffure romantique conforme à l'image de la " bonne dame de Nohant " (ill. 4). Le bas-bleu choque parce qu'elle est une femme contre nature qui a troqué ses vertus féminines contre un cerveau. La femme peut réfléchir mais elle doit conserver sa spécificité féminine dont l'homme a besoin pour se rassurer ${ }^{27}$. 


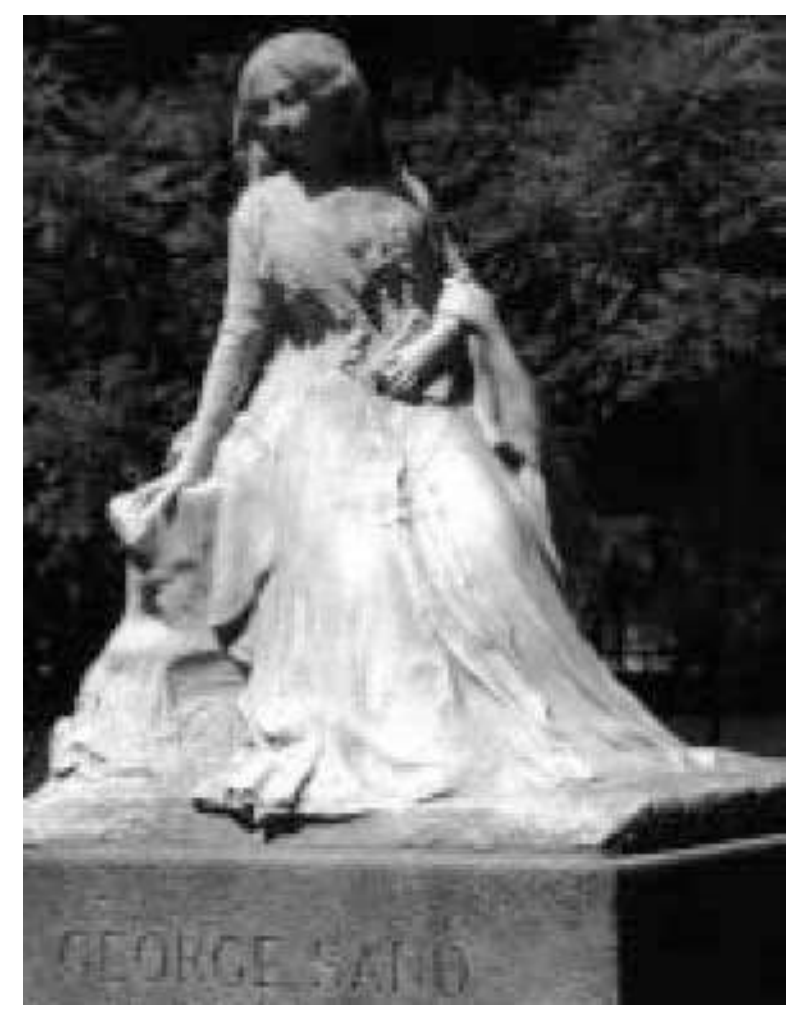

III. 4 - Statue de Georges Sand, Sicard, 1904, jardin du Luxembourg (Tous droits réservés) esprit féminin reste exceptionnel, l'image publique de la femme célèbre ainsi produite demeure relativement conforme aux schèmes de la féminité de l'époque. Cela signifie également qu'on avait bien conscience d'ériger une statue à une femme et non à un grand esprit asexué. Cependant la mention de son nom d'auteur sur le socle participe au processus d'individuation de la femme. On voit écrit publiquement le nom d'une femme qui n'est plus une figuration de la République ou de la Liberté ou le nom d'une reine mais celui d'un individu singulier, différencié. Elle n'est pas saisie à partir de la catégorie globalisante "femme " mais comme un être unique, digne d'admiration et reconnu comme tel. L'étrange singularité de ce prénom masculin sans le « $\mathrm{s}$ » et ce pseudonyme dérivé d'un nom d'homme, Jules Sandeau, seul message sur le socle, témoignait de sa réussite à s'imposer comme actrice, mais signifiait également « la difficulté des femmes à inventer leur propre modèle ${ }^{28} »$.

Le cas le plus exceptionnel est celui de Maria Deraismes, grande théoricienne du mouvement féministe avec Léon Richer, fondatrice et présidente de la Société pour l'Amélioration de la Femme et la Revendication de ses Droits (SAFRD), instauratrice de la mixité dans la franc-maçonnerie ${ }^{29}$. Elle est représentée en habits de l'époque, dans la posture de l'orateur, posture dans laquelle était figuré l'Empereur pendant l'Antiquité. Peu d'hommes célèbres sont représentés de la sorte : cette féministe, célèbre notamment pour les conférences sur des sujets féministes qu'elle donnait, bénéficie d'une représentation inédite, presque incroyable.

UN TRAITEMENT ÉGALITAIRE DES STATUES DE FEMMES CÉLÈBRES ?

27 Les différences de traitements qui existent entre les statues de femmes célèbres sont encore plus nettes si on les compare aux statues des grands hommes. L'analyse du site, de 
la représentation esthétique et de la cérémonie d'inauguration permet de mettre en valeur les similitudes et les différences.

Les signifiants

Le site est souvent choisi à proximité du lieu où a vécu la personne honorée, en fonction des disponibilités. Plus on avance dans le siècle (jusqu'à la fonte des statues par Vichy), moins il reste de sites disponibles. Sachant que certains étaient plus prestigieux que d'autres, les statues de femmes furent-elles placées dans des endroits comparables à celles des grands hommes?

Une même dichotomie semble exister : les statues à caractère politique sont davantage érigées dans l'espace urbain proprement dit, alors que les statues d'artistes et d'hommes ou de femmes de lettres ornent davantage les jardins publics et les squares.

Nous l'avons vu précédemment : de nombreux monuments dédiés à des hommes célèbres sont accompagnés d'une allégorie (une femme souvent nue) placée à ses pieds ou penchée au-dessus de son épaule, qui représente la renommée, l'inspiration, le génie, etc. Comme on peut bien l'imaginer, aucune femme célèbre n'est accompagnée d'une nymphe inspiratrice ou mieux d'un homme nu allongé à ses pieds! Maria Deraismes s'appuie sur une chaise, Mesdames Hirsch et Boucicaut ont à leur pied une femme assise et un petit garçon, tous deux en guenilles : il s'agit de personnages réalistes visant à montrer le caractère charitable des deux grandes dames, rien à voir donc avec une allégorie. Louise Michel est accompagnée d'un petit garçon traité également sur un mode réaliste, placé là afin de montrer avant tout sa charité et son dévouement. Même Louise Michel, dont le projet a avortés ${ }^{30}$, vu le caractère dérangeant du personnage, est traitée de façon à mettre en valeur des qualités spécifiquement féminines. L'inscription sur son socle parle d'ellemême : « À Louise Michel, qui fut la bonté même et ne connut que la misère et la prison ». Louise Michel, la pétroleuse, la pilleuse de boulangeries ${ }^{31}$, celle qui fut déportée et emprisonnée, cette rebelle en armes, s'est muée en femme de bien, sage et dévouée. C'est cette facette de sa vie que l'on a voulu conserver seule, celle de la « vierge rouge » qui donnait tout ce qu'elle possédait aux pauvres et qui éduquait les enfants des rues. À l'époque, Louise Michel pouvait passer pour une dévote, un martyr ${ }^{32}$.

On note également une différence notoire de traitement entre les hommes et les femmes de lettres en ce qui concerne leurs attributs didactiques, éléments qui expliquent au passant la profession du grand homme. Ceux-ci placent toujours les grands hommes pendant l'acte de création : Alexandre Dumas et Voltaire tiennent une plume d'une main et un livre (ou un parchemin) de l'autre, Diderot tient une plume. Or, pour les grandes femmes, leur activité créatrice n'est signifiée que par un livre. Concernant Clémence Royer qui, la première, a traduit Darwin, la substitution peut paraître justifiée. Mais s'agissant de George Sand et de Madame de Staël ${ }^{33}$, les agrémenter d'un livre revient à les placer délibérément dans une posture passive. Elles s'adonneraient, en somme, à une pratique féminine bourgeoise très répandue au XIX ${ }^{e}$ siècle, la lecture, plutôt qu'à une activité créatrice. On affiche donc les hommes comme des créateurs, alors que les femmes de lettres sont représentées comme de simples lectrices. Il en résulte une image somme toute traditionnelle de la femme célèbre, certains blocages culturels empêchant de les traiter sur le même mode que les grands hommes. Cette différence dans le choix des attributs relève de l'inconscient collectif, de schèmes structuraux profondément ancrés dans les mentalités et révèle beaucoup sur la relation entre les sexes. 
32 À regarder de plus près, on voit que cet avènement des femmes de pierre et de bronze dans l'espace public ne se fait pas vraiment sur un pied d'égalité avec les hommes. Elles sont représentées comme des individualités mais on a choisi les facettes les plus conventionnelles de leur personnage comme pour donner une image assagie et rassurante de ces femmes exceptionnelles. Cette image compense la peur née de la figure de la femme excessive, mais également des revendications féministes qui se développent à l'époque et qui renversent les limites assignées à chaque sexe. Seules Maria Deraismes et Jeanne d'Arc font exception. Maria Deraismes apparait comme la grande gagnante de ce peuple féminin. Ni mère, ni furie, ni femme sexuellement libérée, elle échappe aux catégories soit traditionnelles et soit excessives dans lesquelles la femme est enfermée. Elle est représentée sur le même mode qu'un grand homme, tout en conservant une apparence féminine. Sa statue est un véritable hommage public rendu à une femme. Jeanne d'Arc, quant à elle, n'incarne pas une véritable femme et en donne plutôt une image asexuée mais sensible et plurielle.

La construction du sens

33 Le couronnement du processus d'érection d'une statue se joue lors de l'inauguration, moment de liesse, de rassemblement des pouvoirs publics et des citoyens. On donne vie à la statue à travers des discours d'éloge qui consacrent son apothéose. Ils produisent une certaine image de la statufiée, revisitée à l'aune des valeurs des pouvoirs en présence. L'inauguration peut être l'occasion d'un détournement de sens de la statufiée au profit de la glorification narcissique des commanditaires. Dans un second temps, la statue peut enclencher un processus commémoratif, qui tranche, par son aspect informel et spontané, avec le caractère officiel de l'inauguration. Toutes les statues n'ont pas la même efficacité commémorative et n'ont pas suscité des manifestations régulières. Le site de la statue peut devenir un lieu d'affrontement entre différentes factions, instrumentalisé par les oppositions partisanes, comme autour des statues de Jeanne d'Arc entre nationalistes et républicains, ou un lieu de rassemblement pour un groupe minoritaire.

La cérémonie d'inauguration permet des échanges verbaux entre hommes et femmes et donne une visibilité au groupe minoritaire des féministes. Les femmes utilisèrent-elles cette tribune officielle pour clamer leurs revendications ? Afin de répondre à cette question, nous nous attacherons à l'analyse des discours prononcés lors des cérémonies d'inauguration des statues de George Sand et de Maria Deraismes, respectivement le $1^{\text {er }}$ août 1904 et le 3 juillet 1898 .

George Sand, bonne dame romantique ou bas-bleu féministe?

En ce qui concerne le portrait ici tracé de George Sand il faut d'emblée remarquer qu'aucun des hommes ne parle des excentricités qui font d'elle une féministe révoltée, sortie des limites de son sexe. Jules Claretie - président du comité, écrivain et administrateur de la Comédie Française - la présente d'abord comme une mère : il rappelle qu'elle venait avec sa fille Solange dans ce jardin et que jamais elle n'aurait imaginé y voir sa statue, elle qui était si humble, à l'image des autres femmes : " À l'endroit même où George Sand promena ses rêves de jeunesse et ses inquiétudes de mère, Paris acclame aujourd'hui sa gloire, et la postérité salue son image ${ }^{34}$ \%. Les républicains ne peuvent pourtant occulter son engagement en 1848 pour la liberté et la justice sociale. Cependant, son action politique ne viendrait pas d'elle seule, mais de l'influence des hommes. On lui assigne une position complémentaire : "Elle partagea, femme, les espoirs prématurés des hommes illustres qui l'environnaient ». L'image de l'écrivain romantique exaltée, qui effraie quelque peu, est compensée par l'image de la 
mère protectrice, qui s'accorde bien au calme de la statue. George Sand n'est pas traitée sur le même mode que les autres hommes célèbres. Ceux-ci sont en effet souvent présentés comme appartenant à la lignée des grandes figures masculines du panthéon national, comme le montre, par exemple, un extrait de la cérémonie d'inauguration du monument à Delacroix :

Le nom qui brille sous cette fière image est entré dans notre patrimoine commun [... ] Tandis que dans la première moitié du siècle la sculpture aurait cité Rude et Barye, la musique Berlioz, l'histoire Michelet, la poésie Victor Hugo, la peinture, si elle n'avait pas eu Delacroix, fût restée en dehors du mouvement qui transformait le génie français ${ }^{35}$.

Venons-en maintenant aux discours prononcés par des personnalités féministes. Celui de Séverine ${ }^{36}$ est le seul dont nous possédions encore le texte. L'article dit qu'elle l'aurait improvisé en déposant une gerbe, ce qui montre le statut précaire de la parole des femmes dans ce comité où les personnalités officielles masculines interviennent selon un programme prédéfini. On pourrait imaginer un discours glorifiant en George Sand la féministe, l'amazone, le bas-bleu ${ }^{37}$ érudit, celle enfin qui imitait les hommes, s'habillant et fumant comme eux. Or, on est surpris de voir le portrait relativement conventionnel que brosse Séverine de cette femme exemplaire. Elle insiste sur le caractère maternel et protecteur de George Sand. Si les hommes en faisaient la mère des écrivains, les féministes en font la mère et la sœur des femmes qui luttent pour leur reconnaissance, au moyen de leur plume : «Voici tes filles qui t'apportent des roses ».

Vient ensuite un portrait plus novateur de l'écrivain : elle fut " la première professionnelle. [...] Elle s'affirma, en plus qu'une admirable artiste, un probe artisan ; démontra, par l'action, la puissance de travail du cerveau féminin ». On ne glorifie pas tant ici un talent inné que la réussite personnelle de cette femme, grâce à un travail sans relâche. Cet argument vise à démontrer que les femmes sont capables de devenir de grands individus par leur mérite et l'usage de leur cerveau ; il s'oppose à certains discours antiféministes qui affirment l'irréductible infériorité naturelle de la femme. L'usage du terme médical " cerveau » n'est pas anodin. Le cerveau est considéré, au XIX ${ }^{e}$ siècle, comme le « siège des affections nerveuses [...] qui vouent à l'ombre quiète du foyer et exige indulgence (pénale notamment : la femme est quasi irresponsable) et protection ${ }^{38}$ ». Séverine renverse donc ici tous les clichés qui font de la femme un être naturel, déterminé et passif. Par l'action et le travail individuels, une femme peut s'affirmer dans l'espace public et être reconnue. Mais cette affirmation individuelle n'empêche pas de lui reconnaître des valeurs féminines qui la différencient des hommes et qui lui permettent de se poser par rapport à eux (Séverine parle de " cerveau féminin »). Cette phrase reflète significativement la position ambiguë des féministes de l'époque. Celles-ci oscillent entre une affirmation de la femme comme individu, égale de l'homme, et au contraire comme un être différent, spécifiquement féminin. Ces deux arguments, l'un ontologique, l'autre pragmatique coexistent et annoncent cette " citoyenne paradoxale » dont parle Joan Scott ${ }^{39}$. D'un côté, George Sand est l'égale des grands hommes : la journaliste de La Fronde écrit à propos des lettres de George Sand qu'elles doivent être admirées pour " l'éloquence passionnée, la générosité véhémente, la curiosité de mille choses, l'amour de la vie malgré les heures de pessimisme, la sincérité, pour tout ce qu'elles nous apprennent sur une femme de génie et de talent et sur les hommes de talent et de génie qui l'entourèrent et traitèrent avec elle d'égaux à égale ». De l'autre, on reconnaît à George Sand les vertus féminines traditionnelles : «Elle a été la bonne compagne des esprits 
hardis, l'ambulancière qui soulage et réconforte ; qui verse l'héroïsme civique, le philtre de l'enthousiasme, l'ivresse du dévouement au cœur des citoyens!».

Maria Deraismes, révélation des positions féministes

Dans le portrait brossé de Maria Deraismes, grande théoricienne du mouvement féministe, celle-ci est présentée à travers son engagement sous la Commune en tant qu'infirmière dévouée :

Secourable aux blessés, accueillante aux vaincus, ce qu'elle consacra d'intelligence à la défense des petits et des humbles, donnant sans compter et son temps et sa fortune, ce qu'elle dépensa d'efforts pour le triomphe de la République, inséparable à ses yeux de l'idée de justice et de libertét ${ }^{40}$.

Sa charité est donc mobilisée au profit de la glorification de la République : l'inauguration de la statue de Maria Deraismes est vue comme l'occasion « d'exalter dans notre République les idées de justice et de fraternelle égalitét1 ${ }^{\prime 1}$. Non pas que Maria Deraismes ne fût républicaine mais sa tentative de redéfinir les rapports hommes/femmes n'apparaît pas ici au premier plan. On en fait d'abord une grande républicaine afin de mieux célébrer les valeurs du régime en place. Tous les « bons Français » doivent lui être reconnaissants. Après ce portrait qui rattache Maria Deraismes au régime républicain, on aborde le contenu de sa lutte : "Sa passion - et son honneur, - ce fut de lutter avec énergie, par la plume et par la parole, en faveur de l'émancipation de la femme ». Astier ${ }^{42}$ mentionne sa lutte pour ouvrir aux femmes certaines professions et permettre leur participation aux élections des juges des tribunaux de commerce. Voulant prévenir les objections masculines, il comprend pleinement les réserves qu'une telle revendication peut susciter :

Cette thèse a pu paraître dangereuse à des esprits prévenus ou ombrageux ; elle peut, nous en convenons, comporter des réserves et des tempéraments. Mais qui voudrait contester que la tutelle imposée par nos lois et nos mœurs à la femme fût outrageante pour sa dignité et sans profit pour l'état social ${ }^{43}$ ?

Apparaît ici l'idée clé qui explique la position des hommes féministes, selon laquelle la participation de la femme à la société permettrait d'accélérer le progrès social. De même que Stuart Mill avait écrit : " La société est en déficit par la diminution de la femme ${ }^{44}$ ", Montaut - président du comité et de la cérémonie - avance dans son discours l'idée que l'exclusion de la femme " a privé l'humanité d'un précieux élément de progrès, de moralité, de bonheur ». La femme mérite selon lui d'être intégrée à la société : elle a montré ses capacités dans les arts, les sciences, l'industrie et le commerce ; il tente d'inscrire Maria Deraismes dans une lignée de Grandes femmes, moins fournie que celle des Grands hommes et la rapproche des deux esprits féminins les plus célèbres du siècle : Madame de Staël et George Sand.

41 Mais c'est surtout en tant que mère des futurs républicains que la femme apportera quelque chose à la société :

La politique est la résultante de la mise en jeu de toutes les forces sociales ; sentiments, idées, intérêts se combinant, s'organisant en vue d'atteindre un but commun, bien déterminé, le bonheur. Dès lors, si les femmes, les mères, qui jettent les premières semences dans l'intelligence de l'enfant, donnent aux jeunes générations des sentiments, des mœurs et des habitudes démocratiques, il y a de fortes chances pour assurer à l'édifice républicain une indestructible durée ${ }^{45}$.

Le deuxième argument des hommes féministes s'appuie sur le retard de la France par rapport aux autres pays. Cette grande nation ne peut se permettre de sembler rétrograde : le mouvement féministe « honore la France et la rend au moins l'égale à cet égard des 
autres nations européennes ». "Certaines nations ne nous ont-elles pas, du reste, dépassé sans rien avoir à regretter? » La situation de la femme a certes progressé dans la société française, mais elle n'est pas comparable avec celle des femmes d'autres pays ${ }^{46}$.

De cette comparaison, il ressort que le progrès de la condition féminine se développe inéluctablement. On comprend alors l'audace de Paul Brousse ${ }^{47}$ qui, dans son discours, expose avec cynisme ses motivations à se dire féministe : " par pur égoïsme masculin ». Selon lui, le progrès de la condition féminine est irrésistible. À l'image d'une « force de la nature ", il suit un chemin déjà tracé : "La femme s'évade graduellement de sa minorité civile et aboutira assurément un jour à la plénitude de son droit politique ». Autour de la France, « la femme » - catégorie globalisante, comme s'il s'agissait d'un fléau ou d'une conspiration - « réalise graduellement l'œuvre de son affranchissement intégral ». Mais alors que les autres pays, plus éclairés, ont entamé son émancipation progressive, celle-ci risque en France d'arriver sur la scène publique avec la violence d'une révolution. La peur des changements brutaux, mode évolutif propre à la France, est appliquée aux progrès des femmes. Pour éviter une révolution - il parle de " péril féminin » - qui bouleverserait la société dans son entier, ces hommes féministes préfèrent opter pour une méthode réformiste, progressive et modérée. Brousse propose donc en définitive d'accorder l'électorat local aux femmes " pour [les] élever ensuite, bien préparées, à l'exercice du droit politique ».

Il conclut en soulignant cette altérité fondamentale entre les hommes et les femmes : « Tout cela est à vos yeux, Mesdames, une simple restitution légitime, une œuvre d'équité. Aux miens c'est un acte de prévoyance politique ». Afin de maintenir une position dominante, il déconsidère les arguments des femmes, invoquant les principes universels des droits de l'homme : l'égalité et l'équité entre êtres humains. Il réduit leur victoire à une faveur que les hommes leur feraient, par pur utilitarisme. Il ne considère pas en fait cette émancipation comme véritablement légitime ; la nécessité seule le pousse à l'accepter. Cela permet d'inscrire la lutte des femmes en rapport direct avec le pouvoir des hommes qui font " grâce » aux femmes de quelques droits afin de mieux les contrôler et d'empêcher une révolte trop déstabilisante dans cette société bourgeoise et modérée. Il se pose en tant qu'homme et non en tant qu'être humain considérant la femme comme son semblable. Reflet de la crise de l'identité masculine en cette fin de siècle, ce serait donc par peur des débordements féminins que ces hommes auraient accepté d'ériger cette statue à Maria Deraismes et non par reconnaissance de l'action des femmes comme légitime. Maria Deraismes, symbole du féminisme modéré et républicain qui prônait la " politique des petits pas » permet de canaliser la multiplicité des revendications féministes et de les synthétiser en un plaidoyer acceptable.

En définitive, même si l'érection de ces statues de femmes célèbres constitue une première dans l'histoire de l'hommage public parisien, force est de constater que les schèmes de représentations de l'époque régissant les rapports de sexes se retrouvent dans ces effigies inédites. La position des hommes féministes révèle la persistance d'une conception inégalitaire des rapports hommes/femmes et cache une crise de l'identité masculine face à l'émancipation progressive de la femme. Les féministes, quant à elles, auraient pu utiliser cette tribune officielle que constitue la cérémonie d'inauguration où la presse, les hommes politiques et les intellectuels sont présents, pour clamer haut et fort leurs revendications. Une Hubertine Auclert, féministe radicale et première suffragette, prônant un activisme dans la rue, aurait pu y tenter un coup de force. Elle ne l'a pas fait, pourquoi ? Probablement, la retenue bourgeoise l'en a-t-elle empêchée. Les 
revendications des féministes concernent davantage les droits civils de la femme que les droits civiques et politiques. Seule Hubertine Auclert (et quelques autres) demande clairement le droit de vote des femmes. Lorsqu'elle organise une action en 1904 visant à brûler le code civil devant l'Assemblée Nationale, nombre de femmes se dérobent. Cela peut s'expliquer par le fait que les féministes sont des bourgeoises modérées et subissent une forte pression sociale, la femme n'est pas censée prendre part directement à la vie publique et politique. La femme qui sort de sa fonction sociale est considérée comme une prostituée, une folle, une pétroleuse, une tricoteuse, une amazone. Toutes ces images de femmes publiques terrifient les hommes et les femmes elles-mêmes et constituent des contre-exemples. Dans l'ensemble, les inaugurations donnent davantage lieu à des fêtes de souveraineté républicaine et masculines même si les femmes y sont présentes et actives. Leur participation a cependant une portée symbolique majeure en dépit du fait qu'elles n'étaient pas suffisamment mûres pour utiliser la tribune officielle afin d'imposer une image nouvelle de la femme. Les grandes statufiées oscillent entre deux images contrastées : celle de la mère dévouée et protectrice et celle du grand esprit masculin auquel on accole un qualificatif féminin. L'image de la citoyenne est absente de ces catégories stigmatisantes.

\section{BIBLIOGRAPHIE}

AGULHON Maurice, Marianne au combat, l'imagerie et la symbolique républicaines de 1789 à 1880, Paris, Flammarion, 1979. -, Histoire Vagabonde I, Paris, Gallimard, 1988.

BALANDIER Georges, Le Pouvoir sur scènes, 1980, Paris, Balland, 1992.

BRAUD Philippe, L'Émotion en politique, Paris, Presses de Sciences Po, 1996.

CORBIN Alain, « Le secret de l'individu », dans Histoire de la vie privée, t. IV., Philippe Ariès et Georges Duby dir., Paris Seuil, 1987, p. 415-501.

Duby Georges, PERROT Michelle (dir.), L'Histoire des femmes, t. IV, Paris, Plon, 1991. -, Images de femmes, Paris, Plon, 1992.

HARGROVE June, La Représentation des grands hommes dans les rues et les places de Paris, Paris, Albin Michel, 1989.

KLEJMAN Laurence, RochefORT Florence, L'Égalité en marche, Paris, Presses de la Fondation Nationale des Sciences Politiques-Des Femmes, 1989.

MosSE George L., Nationalism and Sexuality, New York, Howard Fertig, 1985.

PERROT Michelle (dir.), Une histoire des femmes est-elle possible ?, Marseille, Rivages, 1984.

\section{NOTES}

*. Cet article est issu d'un mémoire de DEA de Sociologie politique (Université de Paris I) sous la direction de Philipe Braud, soutenu en 1998. 
1.Paris, Bibliothèque de la Pléiade, 1961, p. 1086.

2.D'après Jacques Lanfranchi, Les Statues de Paris, Thèse de 3.cycle, Université de Paris I, 1979 , p. 9.

3. "Pour le bourgeois, hanté par le rôle de héros fondateur, il ne s'agit plus, comme naguère pour l'aristocrate, de s'inscrire dans la continuité des générations, mais de créer une lignée, dont il se doit d'inaugurer le prestige par sa réussite personnelle ». Alain Corbin, « Le secret de l'individu ", dans Histoire de la vie privée, Ph. Ariès et G. Duby (dir.), t. IV, Paris, Seuil, 1987, p. 423.

4.La statue permet d'exprimer un maximum de sens avec le minimum de coût. Cf. E. Sapir, Anthropologie, Paris, Seuil, 1967, p. 50.

5.Voir les travaux de Maurice Agulhon et de June Hargrove.

6.Philippe Braud, L'Émotion en politique, Paris, Presses de Sciences Po, 1996, p. 124. 7.L'analyse des projets avortés révèle avec davantage d'acuité l'enjeu que constitue l'érection d'un monument public.

8.La corbeille des reines de France du jardin du Luxembourg (vers 1848) représente des femmes de rois, des mères de princes. Elles incarnent une lignée aristocratique et un type de régime disparu. Rassemblées dans un même espace de promenade et de loisir, elles avaient davantage une fonction décorative, même si elles annonçaient l'apparition de statues de grandes femmes dans l'espace public.

9. "Tout ou rien, pile ou face, les dichotomies du masculin et du féminin mènent à des impasses. Les zones mixtes, brouillées, les frontières indécises sont plus fructueuses à explorer ». Michelle Perrot (dir.), Une histoire des femmes est-elle possible ?, Marseille, Rivages, 1984, p. 13.

10.Mentionnons également les représentations traditionnelles de la femme, incarnation du sacré comme sainte et comme reine (voir note 8).

11.Voir Maurice Agulhon, Marianne au combat, l'imagerie et la symbolique républicaines de 1789 à 1880, Paris, Flammarion, 1979, p. 233.

12. " Tous ces symboles nationaux et régionaux contribuèrent à fixer la femme à sa place, renforçant encore davantage la différence entre les sexes et par là entre ce que la société bourgeoise percevait comme normal et anormal ». G. Mosse, Nationalism and Sexuality, New York, Howard Fertig, 1985, p. 97.

13.Par exemple les femmes-torches de l'escalier d'honneur de l'Opéra Garnier par Carrier-Belleuse dont les plâtres sont au musée d'Orsay.

14. Méthode pour réaliser une œuvre en pierre à partir d'un original en cire ou argile. Un compas à trois dimensions permet de reporter sur le bloc de pierre les points de repères mis sur le moulage original.

15. À partir d'un modèle en cire, coulé au moyen d'un moule creux, l'ensemble est recouvert de matériaux réfractaires et de tuyaux d'évacuation, puis chauffé. La cire fond, s'écoule, le métal en fusion est introduit par l'intermédiaire des conduits dans l'espace laissé libre.

16.Ces monuments sont érigés au parc Monceau et au jardin du Luxembourg. " C'est un autre aspect fort important de la sculpture au XIX ${ }^{\mathrm{e}}$ siècle que cette obsédante présence du corps féminin. La représentation d'une allégorie, allégorie d'abstraction civique ou morale, d'idéal politique, de nation, de région ou de ville, va devenir presque aussi banale que celle du grand homme. Elle le rejoindra d'ailleurs assez souvent en une même combinaison ; que de grands hommes de bronze seront ainsi guidés par une Victoire, salués par une Renommée, congratulés par une France ou par une Liberté, dressés sur le même piédestal, ou se dressant vers eux à partir d'un étage inférieur du socle ! ", Maurice 
Agulhon, «Imagerie civique et décor urbain dans la France du XIX $x^{e}$ siècle » dans Histoire Vagabonde I, op. cit., p. 112.

17.Ibid., p. 112-113.

18.Article « Femme », dans Grand dictionnaire universel du XIXe siècle, p. 202.

19. « Le corps de la femme atteint un bien moins grand développement que celui de l'homme. Sa constitution corporelle se rapproche de celle de l'enfant ; c'est pourquoi elle est comme celui-ci, d'une sensibilité très vive, se laissant facilement impressionner par les sentiments de joie, de douleur, de crainte, etc. ; et, comme ces impressions agissent sur l'imagination sans être accompagnées d'ordinaire par le raisonnement, il s'ensuit qu'elles sont moins durables et que la femme est plus sujette à l'inconstance », ibid. 20.Les filles sont privées des matières nobles : la philosophie, le latin et le grec et l'enseignement des mathématiques et des sciences naturelles est simplifié. À la place, on leur apprend la couture. À l'issue de leurs études, elles n'obtiennent pas de baccalauréat et ne peuvent donc pas accéder aux universités.

21.Laurence Klejman et Florence Rochefort, L'Égalité en marche, Paris, PFNSP, 1989, p. 15. 22.Louise Michel, Maria Deraismes, Clémence Royer et Madame Boucicaut.

23.Direction des Affaires Culturelles de la Ville de Paris, dossier Clémence Royer.

24.La statue de la place des Pyramides apparaît comme le centre symbolique de l'État et donc de la France. Celle de la place Saint-Augustin est également centrale alors que les deux autres, érigées boulevard Saint Marcel et rue de la Chapelle, ont davantage une fonction de repère local, au cœur d'un quartier de la capitale.

25.George Sand et la comtesse de Ségur sont érigées dans le jardin du Luxembourg, mesdames Hirsch et Boucicaut dans le square du Bon Marché et Maria Deraismes dans le square des Épinettes. On prévoyait d'ériger la statue de Louise Michel place des Abbesses, celle de Clémence Royer devant le Collège de France et celle de Madame de Staël derrière la Madeleine.

26.Ceci s'explique probablement par le fait que sa sœur, qui a commandité la statue au sculpteur Barrias, était assez riche pour financer ce matériau onéreux.

27. «Les plus savoureuses qualités féminines se trouvent souvent annihilées chez le Basbleu; plus de douceur, d'enfantine gaieté, plus d'abandon, plus de souples complaisances, plus de tendresses enveloppantes, plus de ces couvaisons admiratives dont l'écrivain adore l'expression bienfaisante dans son home ", Octave Uzanne, Nos contemporaines. Notes successives sur les Parisiennes de ce temps dans leurs divers milieux, états et conditions, Paris, Libr. Impr. réunies, 1894, p. 174.

28.Mona Ozouf, Les Mots des femmes. Essai sur la singularité française, Paris, Fayard, 1995, p. 178.

29.Elle fut une des premières femmes franc-maçonnes et créa la première loge mixte, l'Ordre maçonnique mixte : le Droit Humain.

30.Archives de Paris : VM 92 (1) Préfecture du département de la Seine, liste des nouveaux monuments pour lesquels des emplacements ont été demandés : 2 femmes sur 18 statues dont aucune n'aboutira, Louise Michel et Mme de Staël.

31.Archives de la Préfecture de Police de Paris : BA 1187, L'Écho de Paris, 28 novembre 1906.

32. Cf. L'Illustration, 22 octobre $1881, \mathrm{n}^{\circ} 417, \mathrm{p} .280$.

33.Cette différence de traitement ne s'explique pas par le fait que les sculpteurs étaient des hommes, puisque la statue de Mme de Staël est sculptée par une femme, Marie-José de Charmoy. 
34.Ce développement s'appuie sur le numéro de La Fronde du $1^{\mathrm{er}}$ août 1904 qui publia les discours de la cérémonie.

35.Archives Nationales : F21 4401, Le Soleil, 7 octobre 1890.

36.Séverine, journaliste, une des rares femmes à s'être fait un nom dans la presse, amie de Marguerite Durand, qui participe à La Fronde.

37.Avant que le terme ne devienne négatif, sous l'effet des discours antiféministes, il pouvait avoir un sens positif : une femme érudite qui réfléchit et écrit.

38.Cf. Michelle Perrot, «Le XIX ${ }^{\mathrm{e}}$ siècle était-il misogyne? ", Les Femmes au pouvoir, dossier de l'Histoire, $\mathrm{n}^{\circ}$ 160, novembre 1992, p. 35.

39.Joan W. Scott, La Citoyenne paradoxale, Paris, Albin Michel, 1998.

40.Bulletin Municipal Officiel : 5 juillet 1898, 17.année, $n^{\circ}$ 180, p. 1887-1888.

41.Ibid.

42.Député et conseiller municipal délégué par la Ville pour recevoir la statue en son nom. 43.BMO : 5 juillet $1898,17^{\mathrm{e}}$ année, $\mathrm{n}^{\circ} 180$, p. 1887-1888.

44.Cité par Eugène Lintilhac, Les Jeudis Littéraires, 18 février 1896.

45.Bibliothèque Marguerite Durand : Dossier Maria Deraismes, discours de Montaut.

46.En France, la femme jouit d'une liberté davantage domestique et professionnelle alors que dans les autres pays européens, elle peut prendre une part active aux institutions locales et même nationales. En Angleterre, elle est électeur et éligible des conseils scolaires et des conseils d'assistance et électeur des conseils municipaux. Dans les pays « neufs ", sa situation est encore meilleure : en Nouvelle-Zélande elle peut devenir député et dans trois provinces des États-Unis elle a pris part à l'élection présidentielle.

47.Membre du comité: Paul Brousse (1844-1912), homme politique, médecin, opposant à l'Empire, membre de l'Internationale, communard, président du conseil municipal en 1905 et député en 1906.

\section{RÉSUMÉS}

Les débuts de la III ${ }^{\mathrm{e}}$ République sont marqués par l'apogée de la statuomanie qui glorifie les Grands hommes et parmi lesquels se distinguent quelques "Grandes femmes ». Cette intrusion dans l'espace public de la femme reconnue comme sujet, qui tranche sur le modèle prédominant de la femme-objet, s'accompagne-t-elle d'une réelle affirmation de la femme comme l'égale de l'homme ? S'il s'agit bien d'une inflexion importante dans l'histoire des représentations du deuxième sexe, l'émergence d'une nouvelle image de la féminité reste prisonnière de blocages culturels communs aux hommes et aux femmes*.

Women, which differences?

ARE FAMOUS WOMEN GREAT MEN LIKE THE OTHERS? A CONTRIBUTION TO PARIS STATUOMANIA

The beginning of the Third Republic is the climax of statuomania which glorifies great men among which a few women distinguish themselves. Is this intrusion in the public space of women acknowledged as being a subject - which contrasts with the predominant model of women as being an object - accompanied by a real affirmation of women as the equal of men? If it actually is an important inflection in the history of the representation of the "Deuxième Sexe ", the 
emergence of a new image of feminity remains imprisonned by a freezing of cultural identity common to men and women.

\section{AUTEUR}

\section{CHRISTEL SNITER}

Christel SNITER, née en 1975, est diplômée de l'IEP de Grenoble, du DESS d'Administration et Gestion de la Musique (Université de Paris IV) et titulaire d'un DEA de Sociologie politique. Elle prépare actuellement une thèse de Science politique à l'Université de Paris I (CRPS), sous la direction de Philippe Braud, sur les statues de femmes érigées à Paris de 1870 à nos jours. c.sniter(at)freesbee.fr 